San Jose State University

SJSU ScholarWorks

Faculty Publications, Chemistry

Chemistry

$1-1-1971$

\title{
Optical Studies of Metal- Semiconductor Transmutations Produced by Intercalation
}

Juana Vivó Acrivos

San Jose State University, juana.acrivos@sjsu.edu

Y. Liang

San Jose State University

J. Wilson

San Jose State University

A. Yoffe

San Jose State University

Follow this and additional works at: https://scholarworks.sjsu.edu/chem_pub

Part of the Physical Chemistry Commons

\section{Recommended Citation}

Juana Vivó Acrivos, Y. Liang, J. Wilson, and A. Yoffe. "Optical Studies of Metal- Semiconductor Transmutations Produced by Intercalation" Journal of Physics C: Solid State Physics (1971): L18-L20.

This Article is brought to you for free and open access by the Chemistry at SJSU ScholarWorks. It has been accepted for inclusion in Faculty Publications, Chemistry by an authorized administrator of SJSU ScholarWorks. For more information, please contact scholarworks@sjsu.edu. 
Garcia-Moliner, F, and Rubio, J., 1969, J. Phys. C: Solid St. Phys, 2, 1789-96.

Morse, P. M. and FeshBACH, H., 1953, Methods of Theoretical Physics, (New York: McGraw-Hill).

\title{
Optical studies of metal-semiconductor transmutations produced by intercalation
}

\begin{abstract}
Spectra of the alkali metal intercalation products of $\mathrm{MoS}_{2}$ and $\mathrm{NbSc}_{2}$ are interpreted in terms of a previously published band model.
\end{abstract}

Studies on the intercalation of the layer type transition metal dichalcogenides with metals and organic materials are of particular interest in connection with the superconducting properties of the more metallic members of this set of compounds. Thus pyridine molecules have been successfully introduced between the sandwiches in niobium and tantalum disulphides and diselenides, and from electrical measurements it is concluded that superconductivity may take place in two dimensions along the sheets (Gamble et al. 1970). The first series transition metals have also been incorporated during vapour transport of $\mathrm{NbSe}_{2}$ elc. into the vacant sites within the van der Waals gap, and some of these products show ordered magnetic states at low temperatures (Anzenhofer et al. 1970, Voorhoeve and Robbins 1970, Hulliger and Pobitschka 1970).

This note is concerned with the optical properties of single crystals of $\mathrm{MoS}_{2}$ and $\mathrm{NbSe}_{2}$ containing sodium intercalated from liquid ammonia. There is already a good deal of structural work on such systems (Rudorff 1965, Omloo and Jellinek 1970). The results obtained from optical measurements can be interpreted in terms of the band model recently developed in publications from this laboratory (for example, Wilson and Yoffe, 1969).

Very thin crystals of $\mathrm{MoS}_{2}$ and $\mathrm{NbSe}_{2}$ about $1000 \AA$ thick were fixed on the window of a small optical cell. The sodium was intercalated between the sandwiches of these small crystals using a refined vacuum microtechnique. $\dagger$ First a film of solid ammonia was deposited onto the crystal, held at $<-78^{\circ} \mathrm{C}$, this being followed by a film of metallic sodium of controlled thickness, produced by directing a beam of sodium atoms onto the cooled crystal. With suitable warming and washing procedures it was possible to progressively 'saturate' the crystal with sodium. The optical absorption spectrum was measured for each sodium concentration in the cell used to prepare the specimen. An absolute measure of the amount of sodium incorporated was not made.

The results for the optical absorption spectra at $77 \mathrm{~K}$ are given in figures 1 and 2 . Figure $1(a)$ shows the spectrum of the pure $\mathrm{MoS}_{2}$ crystal, and figures $1(b)$ and $(c)$ are spectra for the intercalated crystal, containing a small ( 1 to $10 \%$ of saturation limit) and a saturated concentration of sodium respectively. It should be noted that the addition of a small amount of sodium (figure $1(b)$ ) altered the structures C, D and those at higher photon energies more markedly than the exciton peaks A, B; while a saturating concentration of sodium (figure $1(c)$ ) eventually reduced the intensity of all the absorption peaks and gave rise to the characteristic free carrier absorption on the long wavelength side of the absorption edge $(>1 \mu \mathrm{m})$, as for the metal $\mathrm{NbSc}_{2}$ (figure $2(a)$ ). On intercalation of $\mathrm{NbSe}_{2}$ with sodium a peak in optical absorption appears on the main absorption cdge, and the onset of free carrier absorption moves to longer wavelengths. Indeed the spectrum begins to resemble that found for the semiconductors of the $\mathrm{MoS}_{2}$ family - particularly if made p-type by doping with $\mathrm{Nb}$ (see Wilson and Yoffe, 1969, p. 284). If air or water

$\dagger$ For the handling of sodium ammonia solutions see Acrivos and Pitzer (1962). 
2, 1789-96.

(New York: McGraw-Hill).

\section{lutations}

ducts of $\mathrm{MoS}_{2}$ and $\mathrm{NbSe}_{2}$ model.

etal dichalcogenides with on with the superconductmpounds. Thus pyridine dwiches in niobium and urements it is concluded the sheets (Gamble et al. ated during vapour transIs gap, and some of these Anzenhofer et al. 1970 ,

stals of $\mathrm{MoS}_{2}$ and $\mathrm{NbSe}_{2}$ already a good deal of llinek 1970). The results f the band model recently son and Yoffe, 1969).

re fixed on the window of andwiches of these small of solid ammonia was ed by a film of metallic f sodium atoms onto the it was possible to pron spectrum was measured - specimen. An absolute

iven in figures 1 and 2. figures $1(b)$ and $(c)$ are , of saturation limit) and oted that the addition of , D and those at higher le a saturating concentraall the absorption peaks long wavelength side of a)). On intercalation of e main absorption edge. ths. Indeed the spectrum oS. family - particularly , p. 284). If air or water tzer (1962).

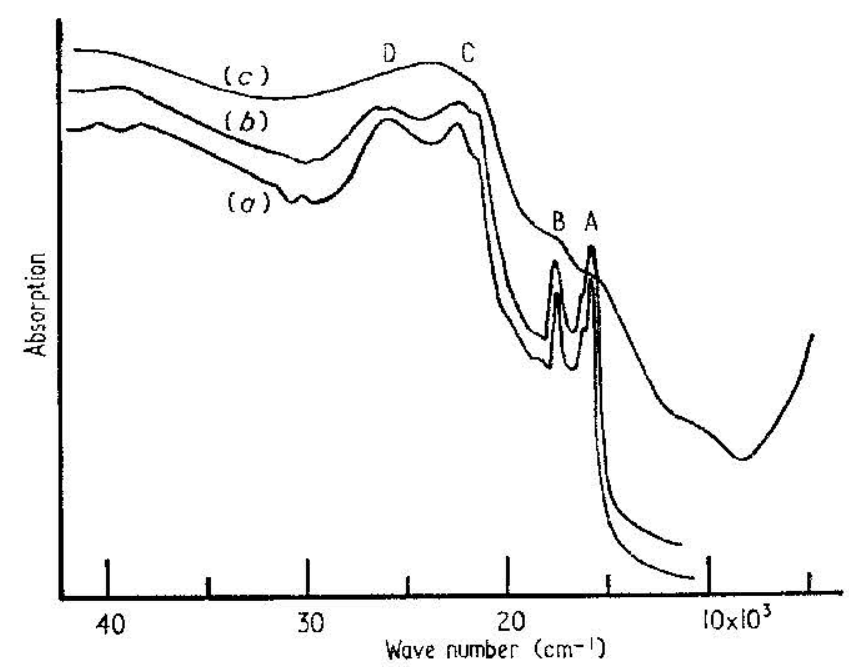

Figure 1. Electronic absorption spectra of cleaved single crystals of $\mathrm{MoS}_{2}$ at $77 \mathrm{~K}$. $(a)$, pure crystal; $(b)$, same crystal intercalated with $\mathrm{Na}$ ( 1 to $10 \%$ of saturation limit); (c), same crystal intercalated with $\mathrm{Na}$ to saturation limit. Curves have been vertically shifted to aid clarity and the absorption coefficients are of order of $10^{5}-10^{6} \mathrm{~cm}^{-1}$ above the band edge.

vapour is gradually admitted into the cell, the sodium reacts and the spectra revert to those of the pure materials, showing the reversibility of the intercalation processes.

If it is assumed that the sodium atoms are ionised in situ $\mathrm{Na} \rightarrow \mathrm{Na}^{+}-+\mathrm{e}$, donating mobile electrons into the $X-\mathrm{M}-X$ sandwiches, $\dagger$ it is possible to explain the experimental results in terms of the rigid band model mentioned in the introduction and illustrated in figure 3.

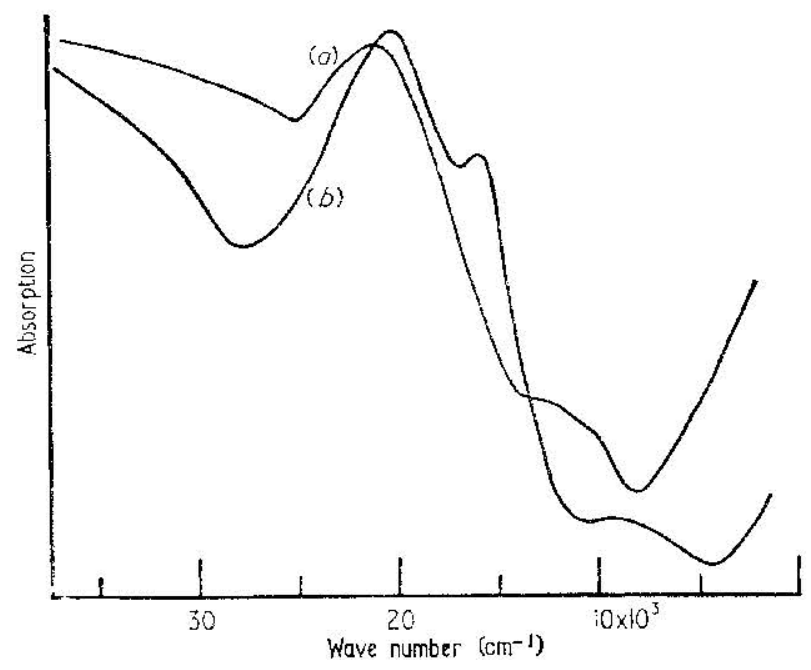

Figure 2. Flectronic absorption spectra of cleaved single crystals of $\mathrm{NbSe}_{2}$ at $77 \mathrm{~K}$. (a), pure crystal; (b), samc crystal intercalated with Na to saturation limit. Absorption coeficients are of order of $10^{5-10^{4}} \mathrm{~cm}^{\cdots 1}$ above the band edge.

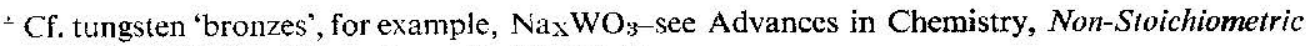
Compounds, 1962 Am. Chem. Soc.,, 39, pp. 224 ff. 
For $\mathrm{MoS}_{2}$, the donated electrons go into the otherwise empty conduction band based on the $\mathrm{d}_{x^{2}-y^{2}}, \mathrm{~d}_{x y}$ orbitals and a metal is formed with the characteristic spectrum shown in figure $1(c)$. The magnetic behaviour changes accordingly from diamagnetism to Pauli paramagnetism (Rudorff 1965). For $\mathrm{NbSe}_{2}$, the extra electrons are introduced into the half filled band based on the $\mathrm{d}_{z^{2}}$ orbital, steadily filling that band. For a fully intercalated

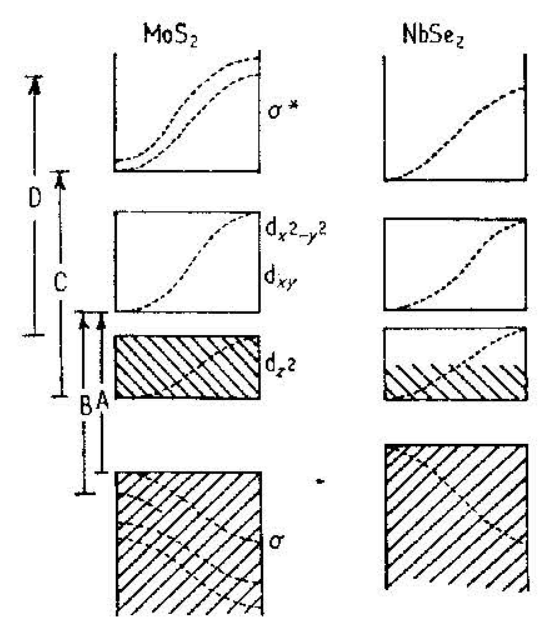

Figure 3. Energy band schemes for $\mathrm{MoS}_{2}$ and $\mathrm{NbSe}_{2}$. Shaded area represents occupied states and the assignment for $\mathrm{A}, \mathrm{B}, \mathrm{C}$ and $\mathrm{D}$ transitions in $\mathrm{MoS}_{2}$ has been indicated.

crystal, namely $\mathrm{NaNbSe}_{2}$, the $\mathrm{d}_{z^{2}}$ band would be complete, the result being a semiconductor akin to $\mathrm{MoSe}_{2}$.

It is intended to make these measurements more quantitative, and also to extend the experiments to include esr, nmr, magnetic, and superconductivity studies.

Cavendish Laboratory, University of Cambridge, Cambridge.

J. V. ACRIVOS $\dagger$ W. Y. LIANG J. A. WILSON A. D. YOFFE 26th October, 1970

Acrivos, J. V., and Pitzer, K. S., 1962, J. Phys. Chem. 66, 1993.

Anzenhofer, K., van Den Berg, J. M., Cossee, P., and Helle, J. N., 1970, J. Phys. Chem. Solids, 31, 1057.

Gamble, F. R., Di Salvo, F. J., Klemm, R. A., and Geballe, J. H., 1970, Science, 168, (3931) 568.

Hulliger, F. and PobitschKa, E., 1970, J. Solid St. Chem., 1, 117.

Omloo, W. P. F. A. M., and Jelliner, F., 1970, J. Less Comm. Metals, 20, 121.

RudorfF, W., 1965, Chimia, 19, 489.

Voorhoeve, J. M. and Robrins, M., 1970, J. Solid St. Chem., 1, 134.

Wicson, J. A. and Yofre, A. D., 1969, Adv. Phys., 18, 193.

† Now at Department of Chemistry, San Jose State College, California, USA.
Nuclear spin
translation of

Abstrac the chat been ste reveals isotropi

Recently (Lenk following depende $\omega_{0}$ (low temperatu.

The value of the e while for the polyi

These results sho stochastic rotation density with $a=2$ Planck equation fo

Torrey (1953) a1 equation for the case, proposed by

$$
J(\omega)=B \tau
$$

with $u=\left(\omega_{0} \lambda\right)^{1 / 2}$.

This relation is s

The nuclear rela to the law $\omega_{0}^{3 / 2}$. Th found for the elas and experimental other than isotrop

On the other ha swollen by $1 \mathrm{cc}$ of explained by the in interproton distan probability of rota exponent $a=2$. $a_{\text {rot }}=2$.

Laboratoire de Sp Faculté des Scienc 38 St Martin d'He

BLOFMBERGEN, N., 1 Hubbard, P. S., 196 